\title{
AN ERGODIC THEOREM FOR FRÉCHET-VALUED RANDOM VARIABLES
}

\author{
D. LANDERS AND L. ROGGE
}

\begin{abstract}
We generalize the classical ergodic theorem from real-valued random variables to Fréchet space-valued random variables and obtain this generalization as a direct corollary of the classical theorem. As an application we obtain several strong laws of large numbers for Fréchetvalued random variables. In a similar way we obtain a martingale theorem for Fréchet-valued random variables.
\end{abstract}

1. Preliminaries. Let $F$ be a Fréchet space and $q_{k}, k \in \mathbf{N}$, be a family of seminorms generating the topology of $F$. Let $(\Omega, Q, P)$ be a probability space.

We shall consider the concept of integration according to Schäfke [4], [5], [6]. Let $\mathscr{F}=\left\{\sum_{\nu=1}^{n} x_{\nu} 1_{A_{\nu}}: x_{\nu} \in F, A_{\nu} \in \mathbb{Q}\right\}$ by the system of $F$-valued simple functions. The integral of a simple function $X=\sum_{\nu=1}^{n} x_{\nu} 1_{A_{\nu}}$ is defined by $E(X)=\sum_{\nu=1}^{n} P\left(A_{\nu}\right) x_{\nu}$.

We can define the integral norm \|\|$:[0, \infty]^{\Omega} \rightarrow[0, \infty]$ (in Schäfke's notation \|\|$_{3}$ ) according to Theorem 5.6.1 of [5]. In our special case of a probability measure this integral norm is given by

$$
\|g\|=\inf \{E(h): g \leqslant h, h \mathbb{Q} \text {-measurable }\},
$$

where $E(h)$ is the classical integral of an $\mathbb{Q}$-measurable function $h$. By

$$
\rho^{*}(X, Y)=\sum_{k=1}^{\infty} \frac{1}{2^{k}} \frac{\left\|q_{k}(X-Y)\right\|}{1+\left\|q_{k}(X-Y)\right\|}
$$

there is defined a pseudometric in the space of all functions from $\Omega$ to $F$. Then a function $X: \Omega \rightarrow F$ is $P$-integrable if there exists a sequence of simple functions $X_{n}: \Omega \rightarrow F$ such that $\rho^{*}\left(X_{n}, X\right) \rightarrow_{n \rightarrow \infty} 0$. Then $E\left(X_{n}\right), n \in \mathbf{N}$, is Cauchy-convergent in $F$ and $E(X)=\lim _{n \rightarrow \infty} E\left(X_{n}\right)$ is the $P$-integral of an $P$-integrable function $X$.

Let $L_{1}(\Omega, Q, P, F)$ be the system of all $F$-valued $P$-integrable functions. According to Theorem 2.4 .5 of [5] we have $X \in L_{1}(\Omega, Q, P, F)$ iff there exists a sequence $X_{n} \in \mathcal{F}, n \in \mathbf{N}$ such that

(1) $X_{n} \rightarrow X P$-a.e.

(2) $E\left(q_{k}\left(X_{n}-X_{m}\right)\right) \rightarrow_{n, m \rightarrow \infty} 0$ for all $k$.

Hence the range of an $P$-integrable function is contained $P$-a.e. in a separable Fréchet space. Let $Q_{0}$ be a sub- $\sigma$-field of $\mathcal{Q}$ and $X \in L_{1}(\Omega, Q, P, F)$. Using

Received by the editors July 6, 1977 and, in revised form, December 12, 1977.

AMS (MOS) subject classifications (1970). Primary 28A65; Secondary 60F15.

Key words and phrases. Pointwise ergodic theorem, strong law of large numbers, weakly orthogonal processes. 
the same techniques as Neveu $[9, \mathrm{p} .100]$ for functions with values in a separable Banach space, one can define the conditional expectation $E\left(X \mid Q_{0}\right)$.

2. The results. We show that the classical ergodic theorem holds true also for Fréchet-valued integrable functions. For the proof of this theorem we only use the classical ergodic theory for real-valued functions. We can immediately derive some strong laws of large numbers for Banach-valued and Fréchet-valued random variables which have been proved before by heavy techniques. With the same technique we show that a martingale theorem for Fréchet-valued random variables holds true. Compare with the heavy techniques used in [1, Satz 22.2] for Banach-valued variables.

THEOREM 1. Let $T$ be a measure preserving transformation of the probability space $(\Omega, \mathbb{Q}, P)$. Let $F$ be a Fréchet space and $X: \Omega \rightarrow F$ be a P-integrable function. Then

$$
\frac{1}{n} \sum_{k=0}^{n-1} X \circ T^{k} \rightarrow E\left(X \mid \sigma_{T}\right) \quad P \text {-a.e. }
$$

where $\mathscr{T}_{T}=\left\{A \in \mathbb{Q}: T^{-1}(A)=A\right\}$ is the $\sigma$-algebra of $T$-invariant sets.

Proof. According to the classical ergodic theorem the assertion is true for all characteristic functions $X=1_{A}$ with $A \in \mathbb{Q}$ and hence for the system $\mathscr{F}$ of all simple functions. Now let $X \in L_{1}(\Omega, Q, P, F)$. Then there exist $X_{j} \in$ $\mathscr{F}, j \in \mathbf{N}$, such that

$$
\rho^{*}\left(X_{j}, X\right) \underset{j \rightarrow \infty}{\rightarrow} 0
$$

and

$$
E\left(X_{j} \mid \mathcal{T}_{T}\right) \underset{j \rightarrow \infty}{\rightarrow} E\left(X \mid \mathcal{T}_{T}\right) \quad P \text {-a.e. }
$$

We have for all $j, k \in \mathbf{N}$ :

$$
\begin{aligned}
& q_{k}\left(\frac{1}{n} \sum_{\nu=0}^{n-1} X\left(T^{\nu}(\omega)\right)-E\left(X \mid \mathcal{\sigma}_{T}\right)\right) \\
& <q_{k}\left(\frac{1}{n} \sum_{\nu=0}^{n-1}\left(X-X_{j}\right)\left(T^{\nu}(\omega)\right)\right)+q_{k}\left(\frac{1}{n} \sum_{\nu=0}^{n-1} X_{j}\left(T^{\nu}(\omega)\right)-E\left(X_{j} \mid \mathcal{F}_{T}\right)\right) \\
& \quad+q_{k}\left(E\left(X_{j} \mid \mathcal{F}_{T}\right)-E\left(X \mid \mathcal{T}_{T}\right)\right) .
\end{aligned}
$$

Since the assertion of the theorem holds for all $X_{j}$ we obtain for all $j, k \in \mathbf{N}$ :

$$
\begin{aligned}
& \varlimsup_{n \rightarrow \infty} q_{k}\left(\frac{1}{n} \sum_{\nu=0}^{n-1} X\left(T^{\nu}(\omega)\right)-E\left(X \mid \mathcal{T}_{T}\right)\right) \\
&<\varlimsup_{n \rightarrow \infty} \frac{1}{n} \sum_{\nu=0}^{n-1} q_{k}\left(\left(X-X_{j}\right)\left(T^{\nu}(\omega)\right)\right)+q_{k}\left(E\left(X_{j} \mid \mathcal{T}_{T}\right)-E\left(X \mid \mathcal{T}_{T}\right)\right) .
\end{aligned}
$$

Let 


$$
f_{j}^{(k)}(\omega)=q_{k}\left(\left(X-X_{j}\right)(\omega)\right)
$$

and

$$
g_{j}^{(k)}(\omega)=\varlimsup_{n \rightarrow \infty} \frac{1}{n} \sum_{\nu=0}^{n-1} f_{j}^{(k)}\left(T^{\nu}(\omega)\right)
$$

By the classical ergodic theorem

$$
g_{j}^{(k)}=E\left(f_{j}^{(k)} \mid \sigma_{T}\right) \quad P \text {-a.e. }
$$

and

$$
E\left(E\left(f_{j}^{(k)} \mid \sigma_{T}\right)\right)=E\left(f_{j}^{(k)}\right) \rightarrow 0
$$

as $j \rightarrow \infty$ by (1). Therefore

$$
g_{j}^{(k)}=E\left(f_{j}^{(k)} \mid \sigma_{T}\right) \rightarrow 0 \quad P \text {-a.e. }
$$

for a subsequence $j \in \mathbf{N}_{0}$. Now the assertion follows from (2) and (3).

Theorem 2. Let $(\Omega, \mathcal{Q}, P)$ be a probability space and $\mathcal{Q}_{n}, n \in \mathbf{N}$, be a sequence of sub- $\sigma$-fields of $Q$ decreasing or increasing to the $\sigma$-field $Q_{\infty}$. Let $F$ be a Fréchet space and $X: \Omega \rightarrow F$ be a P-integrable function. Then

$$
E\left(X \mid \mathscr{Q}_{n}\right) \rightarrow E\left(X \mid \mathbb{Q}_{\infty}\right) \quad P \text {-a.e. }
$$

Proof. According to the classical martingale theorem the theorem is true for all characteristic functions $X=1_{A}$ with $A \in \mathbb{Q}$ and hence for the system $\mathcal{F}$ of all simple functions. Now let $X \in L_{1}(\Omega, \mathcal{Q}, P, F)$. Then there exist $X_{j} \in \mathscr{F}, j \in \mathbf{N}$, such that

$$
\rho^{*}\left(X_{j}, X\right) \underset{j \rightarrow \infty}{\rightarrow} 0 .
$$

We have for all $j, k, n \in \mathbf{N}$

$$
\begin{aligned}
q_{k}\left(E\left(X \mid \mathbb{Q}_{n}\right)-E\right. & \left.\left(X \mid \mathbb{Q}_{\infty}\right)\right) \\
\leqslant & q_{k}\left(E\left(X-X_{j} \mid \mathbb{Q}_{n}\right)\right)+q_{k}\left(E\left(X_{j} \mid \mathbb{Q}_{n}\right)-E\left(X_{j} \mid \mathbb{Q}_{\infty}\right)\right) \\
& +q_{k}\left(E\left(X_{j}-X \mid \mathscr{Q}_{\infty}\right)\right) \\
\leqslant & E\left(q_{k}\left(X-X_{j}\right) \mid \mathbb{Q}_{n}\right)+q_{k}\left(E\left(X_{j} \mid \mathscr{Q}_{n}\right)\right. \\
& \left.-E\left(X_{j} \mid \mathscr{Q}_{\infty}\right)\right)+E\left(q_{k}\left(X_{j}-X\right) \mid \mathbb{Q}_{\infty}\right) .
\end{aligned}
$$

Since the assertion of the theorem holds for all $X_{j}$, we obtain for all $j, k \in \mathbf{N}$ :

$$
\begin{aligned}
\varlimsup_{n \rightarrow \infty} q_{k}\left(E\left(X \mid \mathbb{Q}_{n}\right)-\right. & \left.E\left(X \mid \mathbb{Q}_{\infty}\right)\right) \\
& \leqslant \varlimsup_{n \rightarrow \infty} E\left(q_{k}\left(X-X_{j}\right) \mid \mathbb{Q}_{n}\right)+E\left(q_{k}\left(X_{j}-X\right) \mid \mathbb{Q}_{\infty}\right) .
\end{aligned}
$$

Using the classical martingale theorem we obtain for all $j, k \in \mathbf{N}$ :

$$
\varlimsup_{n \rightarrow \infty} q_{k}\left(E\left(X \mid \mathbb{Q}_{n}\right)-E\left(X \mid \mathbb{Q}_{\infty}\right)\right) \leqslant 2 E\left(q_{k}\left(X_{j}-X\right) \mid \mathbb{Q}_{\infty}\right) .
$$

By (1) for all $k \in \mathbf{N}$ : 


$$
E\left(E\left(q_{k}\left(X_{j}-X\right) \mid \mathbb{Q}_{\infty}\right)\right)=E\left(q_{k}\left(X_{j}-X\right)\right) \rightarrow 0
$$

as $j \rightarrow \infty$. Therefore for each $k \in \mathbf{N}$

$$
E\left(q_{k}\left(X_{j}-X\right) \mid \Theta_{\infty}\right) \rightarrow 0 \quad P \text {-a.e. }
$$

for a subsequence $j \in \mathbf{N}_{1}$. Hence the assertion follows from (2).

It is also possible to obtain in this direct way convergence results for convergence in the $p$ th mean for Theorems 1 and 2.

Considering the canonical process associated to a stochastic process one obtains from Theorem 1 as in the classical case:

COROllaRY 3. Let $X_{n}, n \in \mathbf{N}$, be a stationary process defined on a probability space $(\Omega, Q, P)$ with values in a Fréchet space $F$. Let $X_{1}$ be $P$-integrable, then

$$
\frac{1}{n} \sum_{k=1}^{n} X_{k} \underset{n \rightarrow \infty}{\rightarrow} E\left(X_{1} \mid \mathcal{T}\left(X_{n}: n \in \mathbf{N}\right)\right) \quad \text { P-a.e. }
$$

where $\mathcal{T}\left(X_{n}: n \in \mathbf{N}\right)$ is the system of all invariant sets of the process $X_{n}, n \in \mathbf{N}$.

Since $\mathcal{T}\left(X_{n}: n \in \mathbf{N}\right)$ is contained in the $\sigma$-field of terminal sets of the process $X_{n}, n \in \mathrm{N}$, the zero-one law of Kolmogorov implies that every invariant set has measure 0 or 1 , if $X_{n}, n \in \mathrm{N}$, are independent. Hence we obtain

Corollary 4. Let $X_{n}, n \in \mathbf{N}$, be independent and identically distributed random variables with values in a Fréchet space $F$. Let $X_{1}$ be $P$-integrable then

$$
\frac{1}{n} \sum_{k=1}^{n} X_{k} \underset{n \rightarrow \infty}{\rightarrow} E\left(X_{1}\right) \quad P \text {-a.e. }
$$

Using the fact that a measurable random variable $X$, with values in a separable Fréchet space, fulfilling $E\left[q_{k}(X)\right]<\infty$ for each $k \in \mathbf{N}$, belongs to $L_{1}(\Omega, Q, P, F)$, we obtain the theorem of Taylor and Padgett [7]. Hence we obtain Theorems 4.1.1 and 6.1.2 of [3] as special cases. We can also generalize Theorem 4.3.1 of [3] from Banach spaces with a separable dual to Fréchet spaces with a separable dual.

COROLlARY 5. Let $X_{n}, n \in \mathbf{N}$, be a stationary process defined on a probability space with values in a Fréchet space with a separable dual space. Let $E\left(X_{1}\right)=$ $0, E\left(q_{k}^{2}\left(X_{1}\right)\right)<\infty$ for all $k \in \mathbf{N}$. Let the process be weakly orthogonal, i.e. $E\left(f\left(X_{n}\right) f\left(X_{m}\right)\right)=0$ for all $n \neq m$ and all continuous linear functionals of $F$, then

$$
\frac{1}{n}, \sum_{k=1}^{n} X_{k} \rightarrow 0 \quad \text { P-a.e. }
$$

Proof. According to Corollary 2 we obtain

$$
\frac{1}{n} \sum_{k=1}^{n} X_{k} \underset{n \rightarrow \infty}{\rightarrow} E\left(X_{1} \mid \sigma\left(X_{n}: n \in \mathbf{N}\right)\right) \text {. }
$$


Now using the same method as in $[3$, p. 56] we obtain the assertion.

\section{REFERENCES}

1. M. Metivier, Reelle und verktorwertige Quasimartingale und die Theorie der stochastischen Integration, Lecture Notes in Math., vol. 607, Springer-Verlag, Berlin and New York, 1977.

2. J. Neveu, Discrete parameter martingales, North-Holland, Amsterdam, 1975.

3. W. J. Padgett and R. L. Taylor, Laws of numbers for normed linear spaces and certain Fréchet spaces, Lecture Notes in Math., vol. 360, Springer-Verlag, Berlin and New York, 1973.

4. F. W. Schäfke, Integrationstheorie. I, J. Reine Angew. Math. 244 (1970), 154-176.

5. __ Integrationstheorie. II, J. Reine Angew. Math. 248 (1971), 147-171.

6. __ Integrationstheorie und quasinormierte Gruppen, J. Reine Angew. Math. 253 (1972), 117-137.

7. R. L. Taylor and W. J. Padgett, Some laws of large numbers for normed linear spaces and Fréchet spaces (to appear).

Mathematisches Institut der Universität Köln, 5 Köln 41, Weyertal 86-90, West Germany

Fachbereich Statistik, Universität Konstanz, Postfach 7733, D-7750 Konstanz, West GermanY 